\title{
Specialist and Generalist Services
}

\section{Impact of Generalist Care Managers on Patients with Diabetes}

\author{
David A. Dorr, Adam Wilcox, Steven M. Donnelly, Laurie Burns, \\ and Paul D. Clayton
}

Objective. To determine how the addition of generalist care managers and collaborative information technology to an ambulatory team affects the care of patients with diabetes.

Study Setting. Multiple ambulatory clinics within Intermountain Health Care (IHC), a large integrated delivery network.

Study Design. A retrospective cohort study comparing diabetic patients treated by generalist care managers with matched controls was completed. Exposure patients had one or more contacts with a care manager; controls were matched on utilization, demographics, testing, and baseline glucose control. Using role-specific information technology to support their efforts, care managers assessed patients' readiness for change, followed guidelines, and educated and motivated patients.

Data Collection. Patient data collected as part of an electronic patient record were combined with care manager-created databases to assess timely testing of glycosylated hemoglobin (HbA1c) and low-density lipoprotein (LDL) levels and changes in LDL and HbAlc levels.

Principal Findings. In a multivariable model, the odds of being overdue for testing for HbA1c decreased by 21 percent in the exposure group $(n=1,185)$ versus the control group $(n=4,740)$. The odds of being tested when overdue for HbA1c or LDL increased by 49 and 26 percent, respectively, and the odds of HbA1c $<7.0$ percent also increased by 19 percent in the exposure group. The average HbA1c levels decreased more in the exposure group than in the controls. The effect on LDL was not significant.

Conclusions. Generalist care managers using computer-supported diabetes management helped increase adherence to guidelines for testing and control of $\mathrm{HbAlc}$ levels, leading to improved health status of patients with diabetes.

Key Words. Patient care management, chronic illness, diabetes mellitus, medical informatics

Diabetes mellitus and its complications comprise one of the most expensive categories of chronic disease in the United States, contributing to at least 213,062 deaths in 2000 and $\$ 132$ billion in costs in 2002. There is significant 
potential for improvement when appropriate medical care is provided (American Diabetes Association 2003). The highest potential for improvement comes from the capability to prevent the deadly complications of this disease; careful control of blood pressure, control of glycoslylated hemoglobin (HbAlc) and low density lipoprotein (LDL) level, and administration of appropriate medications (including ACE inhibitors, statins, aspirin, and $\beta$-blockers) have been shown to slow, and, in many cases, stop the progression of microvascular disease in people with diabetes (Matthews 1999; Nicollerat 2000).

However, the United States' success in achieving tight control of HbAlc levels and appropriate medication administration in these patients has been limited at best (Toth et al. 2003). Despite implementation efforts at over half of the major health systems in the United States, compliance with management guidelines remains low. In a recent study, only 10.4 percent of patients met HbA1c, blood pressure, and LDL goals and only 13 percent met medication standards after guideline implementation (Toth et al. 2003). Clearly, people with diabetes and those caring for them have difficulty adhering to these guidelines.

Guideline compliance can be increased through improved processes of care or disease management. One heavily studied approach involves an additional team member called a care manager who facilitates changes in clinic processes and patient knowledge and behaviors. Several studies have shown that interventions involving care managers can help patients and other care providers improve the quality of care and outcomes in diabetes (Pan et al. 1997; Tuomilehto et al. 2001; Knowler et al. 2002; New et al. 2003; Taylor et al. 2003; The California Medi-Cal Type 2 Diabetes Study Group 2004) and other diseases (Bond et al. 1988; Allen 1994; McGrew et al. 1995; Crystal, Lo Sasso, and Sambamoorthi 1999; Naylor et al. 1999; Bull, Hansen, and Gross 2000).

These studies focus almost entirely on specific diseases or conditions and are mostly efficacy-style trials of disease management, or "a coordinated system ... for a specific chronic illness" (AHM 2001), as opposed to a more

Address correspondence to David A. Dorr, M.D., M.S., Department of Medical Information \& Clinical Epidemiology, Oregon Health \& Science University, 3181 SW Sam Jackson Park Road, Mailcode: BICC; Portland, OR 97239. Adam Wilcox, Ph.D., and Paul D. Clayton, Ph.D., are with the Department of Medical Informatics, University of Utah, Salt Lake City, UT, and are also with Intermountain Health Care, Salt Lake City, UT. Steven M. Donnelly, Ph.D., is with HealthInsight, Inc., in Salt Lake City. Laurie Burns, P.T., M.S., is with Intermountain Health Care in Salt Lake City. 
broadly defined vision of care management, e.g., "a collaborative process of assessment, planning, facilitation and advocacy" (CMSA 2003). Disease management programs frequently create specialized clinics, which represent a highly focused setting where providers have in-depth training in a single disease whether they are specialists or trained primary care providers. In these specialized clinics or disease-specific clinic sessions, processes can be more easily controlled than in a general clinic where a multitude of acute and chronic illnesses are treated. In contrast, we studied the impact when care management was used to help treat a patient population with multiple chronic and acute illnesses and needs; care management was characterized by generalist care managers and specially developed information technology to support collaboration during the general primary care workflow. As persons with multiple chronic illnesses are known to suffer higher rates of complications and mortality, the generalist approach has the theoretical advantage of treating the whole person with one or more chronic disease rather than focusing on one disease (Rothman and Wagner 2003; Norris and Olson 2004). In practice, however, this approach is challenging. One study in which a broader patient population was treated demonstrated increases in adherence to guidelines and patient satisfaction, but did not find reductions in HbAlc in the patients with diabetes (Wagner et al. 2001). In other studies, it was found that care management programs increased the use of resources (D'Ercole et al. 1997). This finding is of special concern for overworked primary care clinics that frequently only receive a fraction of the savings that result from improving the health of their patients (Casalino 2003). Thus, it is important that implementation of such programs be carried out carefully, especially in real-world settings with diverse patient populations and limited resources.

Given these concerns, we hypothesized that specialized care could be generalized into a multidisease care management model. To do so, we implemented Wagner's Chronic Care Model (CCM) (Bodenheimer, Wagner, and Grumbach 2002a, b) in a way different from many of the previous studies. At Intermountain Health Care (IHC) in Salt Lake City, we adopted a team approach (with the patient at the center) to encourage patient self-management and improved connection to community resources, and created core health care organization goals as part of a model to improve the care of chronic illness; these interventions are all standard parts of Wagner's CCM. Two major capabilities from the CCM were implemented to address the need to integrate the care management program into primary care workflow. Care managers were placed in the clinics and trained to facilitate team collaboration and general patient education, a more central role than advocated for in the 
CCM. In addition, existing information technology was leveraged and new applications were created to enable the primary care teams (including the care manager) to adopt many different guidelines at once. We hypothesized that the use of computerized alerts, summarized patient information, and electronic communication would allow an integrated approach to successfully meet the needs of patients with chronic illnesses without the need for specialized clinics for each disease or patient population. This information technology would aid the care manager, who would also work with the patient to assess their readiness to change and create a specific care plan based on any of the patient's particular chronic illness(es) (Spencer et al. 2002; Duran 2003). The generalist care manager, with support of the information system, can then act as a catalyst in each clinic, creating and then helping enact the care plan with the patient.

We also hypothesized that the care of patients with diabetes would especially improve in our multidisease, collaborative care management model as patients with diabetes have a very high rate of co-occurring conditions that can worsen disease outcomes (Rothman and Wagner 2003). Improvement was measured by assessing changes in processes (such as timely testing for disease markers), and outcomes (changes in the levels of these markers indicating control) as defined by current diabetes guidelines (AACE 2000; ADA 2003; Goldstein et al. 2004; Haffner 2004). The demands and benefits of successful multidisease care management programs that can be implemented in the workflow of primary care clinics need to be defined, especially in diseases where they have the most impact. When one attempts to integrate multidisease care management systems into primary care, one may dilute the benefit, that might accrue to patients who are treated in a specialized setting. Integrated care management systems offer the promise to improve quality in a cost-effective manner. By examining the changes in adherence and outcomes in a generalist implementation within diabetes, we hoped to determine whether positive effects can be substantial when examining the impact on a single disease.

\section{METHODS}

\section{Health Care Organization}

IHC is an integrated delivery network consisting of 20 hospitals and more than 1,200 employed and affiliated physicians in Utah and Idaho. The 450 physicians employed by IHC work in one of 92 clinics, and provide for more than 
three million outpatient visits each year. For this study, IHC augmented the services of selected primary care providers in seven IHC-owned ambulatory clinics by installing care managers on-site and adding specific information technology. On average, each care manager serves as a resource to 6 to 10 primary care physicians and has a panel of 350 to 500 active patients. Care managers are trained professionals; all seven in this study were either registered nurses or social workers. Four similar reference clinics without care managers, but serving a similar patient population, were used to generate a control population. The control clinics were matched on provider type and experience, staffing, and variety of patient conditions. This study was approved by the local Institutional Review Board as meeting the criteria for ethical human subjects research.

\section{Care Delivery Design and Information Technology}

Exposure to the intervention was defined as referral to, and at least one visit with, a generalist care manager who adhered to the care management delivery model, and used the advanced information technology applications. Patients were referred to care managers by primary care physicians at the physicians' discretion; the providers were encouraged to refer when the patient or their family needed education, cognitive, and community/social support to deal with illness. Referral was not based on specific criteria as perceived need was felt to be the most inclusive indicator for the effectiveness study. For instance, only a subset of patients with diabetes are sent; reasons for referral range from out-of-control glucose levels to those with complicating conditions (e.g., nonsupportive home environment).

Once a patient is referred, the care managers offer all pertinent services to the patients and their families, regardless of diagnosis. The general care management program of which they are a part has several components. With referral, a care management team is activated with the care manager acting to provide continuity, regular follow-up, and collaboration. The care managers meet initially with patients, providing education for disease-specific and general problem-solving skills, motivation to encourage self-management, and development of care specific plans, which frequently include several diseases. The self-management component is facilitated by a care manager assessing the patients' readiness to change to self-managing behaviors, providing ongoing motivation/feedback, and encouraging patient independence, usually through a series of phone calls to patients. The care managers put the patients and their caregivers in touch with community resources and advocate for the patient 
within and beyond the immediate care team both in person at case conferences and via the phone.

Substantial informational technology access was given to all team members, whether care managers were involved or not. The information technology provided Access to patient information, provided reminders and structures for Best practices, and enabled virtual Communication. For Access, team members have access to a longitudinal electronic health record (EHR). The EHR includes the option to use a summarized patient worksheet for chronic diseases. The patient-specific electronic summary gives an overview based upon the chronic conditions of the patient. Team members have access to computer alerts (such as drug-drug interactions) and chronic disease reminders on the summarized form to help support Best practices. The logic in guidelines is extracted in order to generate reminders automatically either via active alerts or on the patient summary as passive prompts.

For the exposure group only, care managers have an additional alerting system that reminds them of specific process-based tasks to perform, such as calling a set of people with diabetes when their tests are overdue. The care management system also has a specific interface that allows care managers to store and retrieve information specific to their workflow. For example, a phone contact for depression has coded elements that easily link to standardized mental health forms.

Finally, all team members have access to an electronic Communication system that allows providers to exchange electronic messages that are ultimately attached to a specific patient's chart. As both control and intervention patients cared for in this study have providers who have the option to use the clinical information system, the information technology portion of the intervention is restricted to the specific care management components and the activities of the care managers themselves.

The diabetes-specific component of this intervention is two-fold. First, all team members are trained in several chronic disease guidelines further developed by IHC from national sources, including ones for diabetes and hyperlipidemia (AACE 2000; ADA 2003; Goldstein et al. 2004; Haffner 2004). From these guidelines, specific diabetic reminders are built into the general information system in the summarized, structured form. In addition, tickler lists in the care manager application display lists of patients who need followup calls for missed tests and patients with high test values. Another aspect of the care model is the specific diabetes education provided by care managers; although two of the seven are Certified Diabetes Educators, all are trained in basic diabetes education. 


\section{Sample Size and Eligibility}

For the purposes of this study, a diabetes registry containing 25,273 patients (as shown in Figure 1) was created by analyzing data from patients seen in the seven care manager clinics and four control clinics. The diabetes registry was created by identifying patients with two or more separate ambulatory visits within the 5-year period between January 1, 1997 and August 1, 2002 with an ICD-9 code of 250. $x x$ (where $x x$ indicates a subdiagnosis of diabetes). Patients were assigned to the exposure group if they had had any encounter with a care manager from March 1, 2001 to September 30, 2002 (the study period). This criterion produced a total of 1,185 exposure patients, who were seen in seven clinics and were co-managed by any of 65 physicians and 7 care managers. Of the 24,088 patients remaining who did not see a care manager, 9,813 had no outpatient encounters during the study period and were excluded. The remaining patients $(n=14,275)$ were used to match control patients in a $4: 1$ ratio $(n=4,740$ matches); clinics with and without care managers contributed similar numbers of controls.

The exposure group start date was defined as the first outpatient encounter with a care manager during the study period, and the start date of the

Figure 1: Sample Size and Eligibility

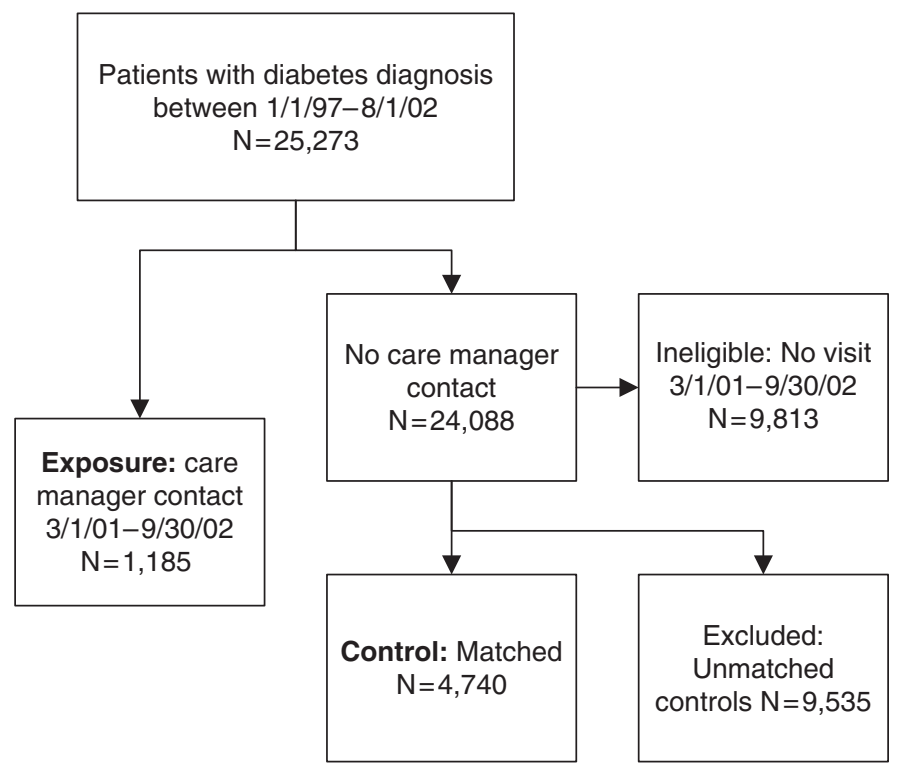


control group as the first outpatient encounter during the study period in which diabetes was included on the diagnosis list. Follow-up time, which began accruing after the individually defined start date, ranged from 4 to 18 months.

\section{DESIGN}

The study design was a retrospective cohort design with matched controls in a 4:1 ratio. Each case was matched to four controls by sex, age, a comorbidity index, the testing regularity pattern (regular, irregular, no testing, or unknown), and previous pattern for glycemic control (controlled, uncontrolled, or unknown) of LDL or HbA1c. Ages were grouped in 10-year intervals based on clinically significant formulations from previous studies (Turner et al. 1999; Mokdad et al. 2003; Engelgau et al. 2004). Regularity and control definitions were based on patient data during the 2-year period prior to the start date, and are described in Table 1. The target goal for desirable HbAlc levels changed from the eligibility period (2001-2002) to the study period (2002-2003) from 7.2 to 7.0 percent; this change is reflected in the differences between baseline control and study control definitions in Table 1 . The comorbidity index was based on the work by Deyo, Cherkin, and Ciol (1992). In their approach, the co-existing diseases in a single patient during the baseline period (represented by ICD-9-CM codes from outpatient visit billing codes) are weighted and summed, with a maximum score of 14 comorbidities. This scale was collapsed into three categories $(1,2$, or 3 or more comorbidities) for matching and data analysis purposes.

\section{Outcome Measures}

Outcomes were process and health status indicators as shown in Table 1. Process variables were adherence to established diabetic and hyperlipidemia guidelines, including the conformity to testing frequency. Use of information systems by the care managers was assessed by audit trails and self-report. Beginning from the treatment initiation date, automated retrospective analysis was carried out for each individual to determine whether laboratory tests were current or overdue based on agreed-upon standards of care and whether observed laboratory test values fell above or below a desired threshold. Patients were overdue for testing if 7 months (for HbA1c) or 13 months (for LDL) had elapsed since the last abnormal test. The desired guideline thresholds were set at $\mathrm{HbA1c} \leq 7.0$ and $\mathrm{LDL} \leq 100$ during the period demarcated by 
1408 HSR: Health Services Research 40:5, Part I (October 2005)

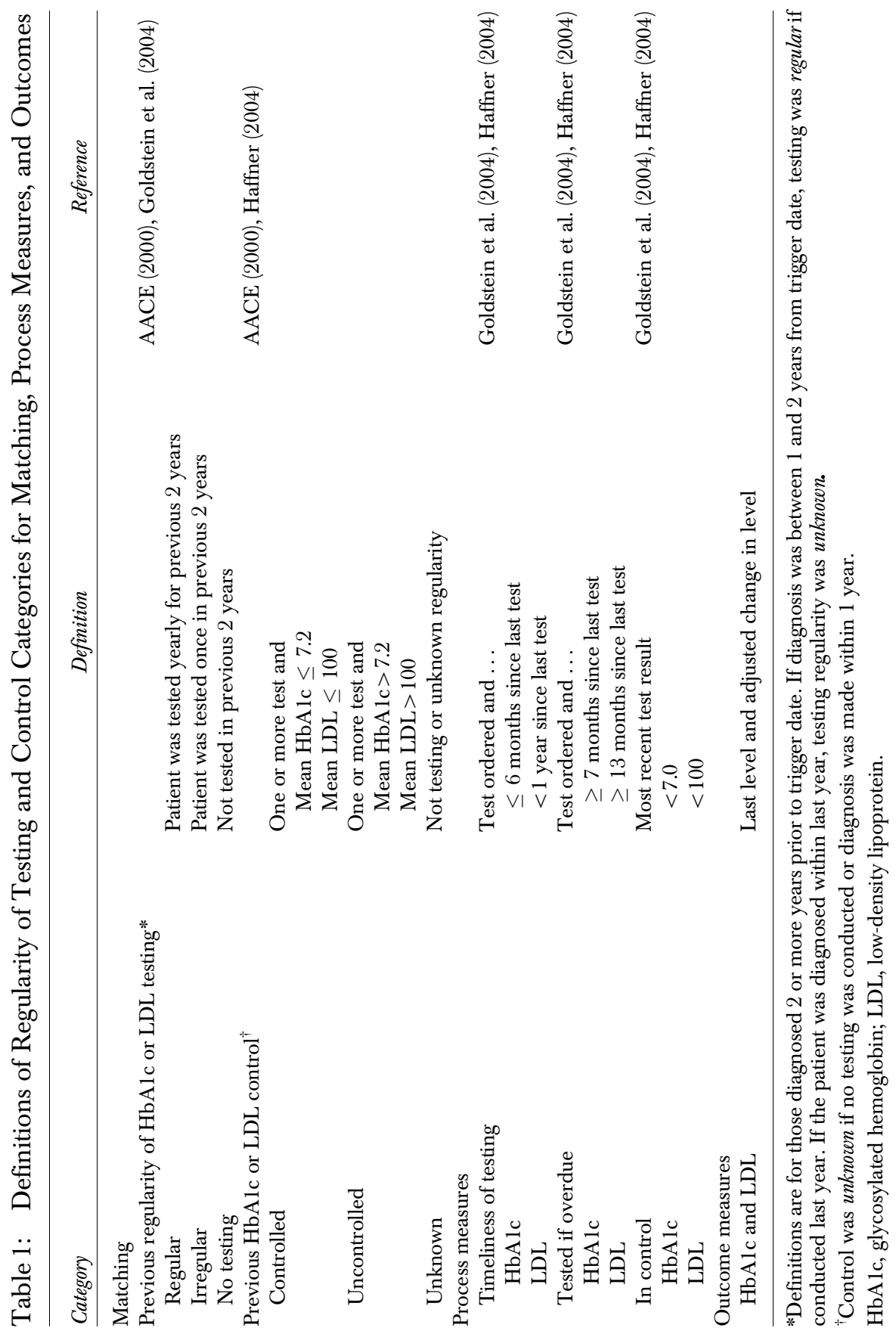


this study. Health status outcome indicators were the levels of both HbAlc and LDL.

\section{Statistical Analysis}

The effects of generalist care managers on outcomes were assessed using logistic and linear regression. Estimates for the main effect of care management were adjusted for patient age (in 10-year age categories), sex, comorbidities, history of testing regularity, race, and history of $\mathrm{HbAlc}$ and $\mathrm{LDL}$ control. The monthly snapshots of the data presented the potential for each patient to have multiple observations, so variance estimation techniques clustered on patient identifier were used to correct for the effects of multiple observations (Huber 1967; White 1982). Although patients were matched on previous control of diabetes (see Table 1) as measured by HbA1c level at baseline, they were not matched on exact HbA1c levels as it was thought that this would lead to overmatching. Differences in baseline levels and subsequent changes were adjusted for possible regression to the mean using the method of Trochim (2003). In this conservative adjustment, intraindividual correlations $(r)$ of change are used to estimate the proportion of change in $\mathrm{HbAlc}$ levels that may be because of statistical artifacts.

\section{RESUlTS}

During the study period, 4,421 patients were referred to seven care managers by 65 physicians; of these referrals, 1,185 (26.8 percent) had diabetes and were assigned to the exposure group. From patients with diabetes seen by physicians and not care managers, 4,470 controls were matched to the study subsets via the criteria described in the methods. The demographic information for the exposure, control, and eligible registry patients is displayed in Table 2. For the unmatched categorical variables (including race), the exposure group distribution was not significantly different from the control one. As an entire group, the registry patients were different from exposure and control groups in that they had a slightly higher disease burden (29.7 percent with two or more diseases versus 26 percent for the other two groups), and had significantly less follow-up and thus more missing information.

Care managers had encounters with patients an average of $4.5 \pm 1.8$ times per 1 year of follow-up. Diabetes was the most frequent reason for referral (26.8 percent), followed by mental health (24 percent), and resource assistance (12 percent) needs. Comparing patients referred for diabetes with others in the care 
Table 2: Group Baseline Characteristics

\begin{tabular}{|c|c|c|c|c|c|c|c|}
\hline & & \multicolumn{2}{|c|}{ Exposure } & \multicolumn{2}{|r|}{ Control } & \multicolumn{2}{|c|}{ Registry } \\
\hline & & $N$ & $\%$ & $N$ & $\%$ & $N$ & $\%$ \\
\hline \multicolumn{2}{|l|}{ Total } & 1,185 & 100.0 & 4,740 & 100.0 & 14,275 & 100.0 \\
\hline \multicolumn{2}{|l|}{ Female } & 603 & 50.9 & 2,412 & 50.9 & 7,170 & 50.2 \\
\hline \multicolumn{8}{|l|}{ Age } \\
\hline \multicolumn{2}{|l|}{$18-29$} & 52 & 4.4 & 208 & 4.4 & 787 & 5.5 \\
\hline \multicolumn{2}{|l|}{$30-39$} & 74 & 6.2 & 296 & 6.2 & 929 & 6.5 \\
\hline \multicolumn{2}{|l|}{$40-49$} & 199 & 16.8 & 796 & 16.8 & 2,049 & 14.4 \\
\hline \multicolumn{2}{|l|}{$50-59$} & 264 & 22.3 & 1,056 & 22.3 & 3,053 & 21.4 \\
\hline \multicolumn{2}{|l|}{$60-69$} & 296 & 25.0 & 1,184 & 25.0 & 3,249 & 22.8 \\
\hline \multicolumn{2}{|l|}{$70-79$} & 219 & 18.5 & 876 & 18.5 & 2,995 & 21.0 \\
\hline \multicolumn{2}{|l|}{$80+$} & 81 & 6.8 & 324 & 6.8 & 1,213 & 8.5 \\
\hline \multicolumn{2}{|l|}{ Mean $(\mathrm{SD})$, years } & \multicolumn{2}{|c|}{$59.9(15)$} & \multicolumn{2}{|c|}{$59.8(15)$} & \multicolumn{2}{|c|}{$60.1(16)$} \\
\hline \multicolumn{8}{|l|}{ Race* } \\
\hline \multicolumn{2}{|c|}{ American Indian } & 2 & 0.2 & 11 & 0.2 & 28 & 0.2 \\
\hline \multicolumn{2}{|c|}{ Asian/Pacific Islander } & 22 & 1.9 & 111 & 2.4 & 301 & 2.1 \\
\hline \multicolumn{2}{|l|}{ Black } & 9 & 0.8 & 30 & 0.7 & 123 & 0.9 \\
\hline \multicolumn{2}{|l|}{ Hispanic } & 67 & 5.7 & 15 & 6.4 & 918 & 6.4 \\
\hline \multicolumn{2}{|l|}{ Unknown } & 26 & 2.2 & 106 & 2.2 & 1,268 & 8.9 \\
\hline \multicolumn{2}{|l|}{ Caucasian } & 1,059 & 89.4 & 3,718 & 88.5 & 11,637 & 81.5 \\
\hline \multicolumn{8}{|l|}{ Risk score } \\
\hline \multicolumn{2}{|l|}{1} & 867 & 73.2 & 3,468 & 73.2 & 10,031 & 70.3 \\
\hline \multirow{4}{*}{$\begin{array}{l}2 \\
3+\end{array}$} & & 264 & 22.3 & 1,056 & 22.3 & 3,717 & 26.0 \\
\hline & & 53 & 4.5 & 212 & 4.5 & 527 & 3.7 \\
\hline & & posure & & Contr & & $\operatorname{Reg} i$ & \\
\hline & $H b A 1 c(\%)$ & $L D L(\%)$ & & $1 c(\%)$ & $L D L(\%)$ & $H b A 1 c(\%)$ & $L D L(\%)$ \\
\hline \multicolumn{8}{|l|}{ Testing history } \\
\hline Unknown & 0.9 & 6.9 & & .9 & 6.9 & 8.5 & 15.2 \\
\hline Not tested & 11.5 & 11.2 & & 1.5 & 11.2 & 11.9 & 19.8 \\
\hline Irregular & 34.3 & 32.1 & & 4.3 & 32.1 & 28.2 & 31.8 \\
\hline Regular & 53.2 & 49.8 & & 3.2 & 49.8 & 51.4 & 33.1 \\
\hline Control history & & & & & & & \\
\hline Unknown & 11.8 & 17.6 & & 1.8 & 17.6 & 20.2 & 34.4 \\
\hline Uncontrolled & 44.6 & 40.3 & & 1.6 & 40.3 & 45.3 & 34.7 \\
\hline Controlled & 43.6 & 42.2 & & 3.6 & 42.2 & 34.6 & 30.9 \\
\hline
\end{tabular}

*Race was not a matching variable; the race distribution between control and exposure groups was not significantly different.

HbAlc, glycosylated hemoglobin; LDL, low-density lipoprotein.

management group, the patients with diabetes had more visits than care-managed patients with other diagnoses, or $5.8 \pm 2.0$ visits per year. In all, there were 6,876 visits completed by care managers for patients with diabetes; 39.4 percent 
were via phone, 36 percent were visits with the patient, 11.9 percent were care conferences or other advocating activities, and 5.5 percent were in a group education session. Seventy percent of all encounters in patients with diabetes involved diabetic education or protocol adherence checks; 14 percent of encounters were for financial assistance with medications, and the remaining 16 percent of encounters in patients with diabetes were solely for other diseases, including depression, hypertension, and drug dependency. During the study period, care managers addressed at least one other major issue besides diabetes in 35 percent of patients with diabetes. Care managers accessed the electronic records of every patient at least once during the study period, using the computer to see laboratory and radiology test results, to read physician progress notes, or to review measures of chronic disease adherence on the patient worksheet. Best practice support provided by the patient worksheet or tickler lists to remind care managers of follow-up appointments were used daily by three (of seven) of the care managers, used weekly by three additional care managements, and used at least monthly by all seven. In addition to phone calls, communication among team members via the electronic messaging system was used by care managers at twice the rate of physicians per patient seen; as their receipt of messages was higher, physicians saw 29 percent more electronic messages in the care of exposure patients $(1.0 \pm 3.7$ messages per patient $)$ versus controls $(0.7 \pm 2.1$ messages per patient). Physicians of control patients used the information system on 93 percent of all patients, including alerts, the summarized worksheet, and communication between providers about patient status. Beyond the care manager-specific applications and message log use, no significant difference in information system use by physicians was seen in the care of exposure versus control patients.

Table 3 shows the unadjusted and adjusted odds ratio (OR) for the exposure group versus the control group in adherence to the diabetes guidelines. Before adjustment for other variables, the patients in the exposure group had 20 percent lower odds of being overdue for HbA1c testing, were 42 and 20 percent more likely to be tested for HbAlc and LDL if overdue, and were 24 percent more likely to have an HbA1c under the goal threshold of 7.0. All of these values were significant at the $p<.01$ level in both the single and multivariable model except LDL testing when overdue $(p=.10$ for single and $p=.04$ for multivariable models).

In the multivariable model, the exposure group was 21 percent less likely to be overdue for $\mathrm{HbAlc}$ testing (OR 0.79, 95 percent confidence interval [CI] 0.72-0.85), and 31 percent more likely to have an HbAlc under 7.0 percent, as shown in Table 3 . Also significant in the model were testing 


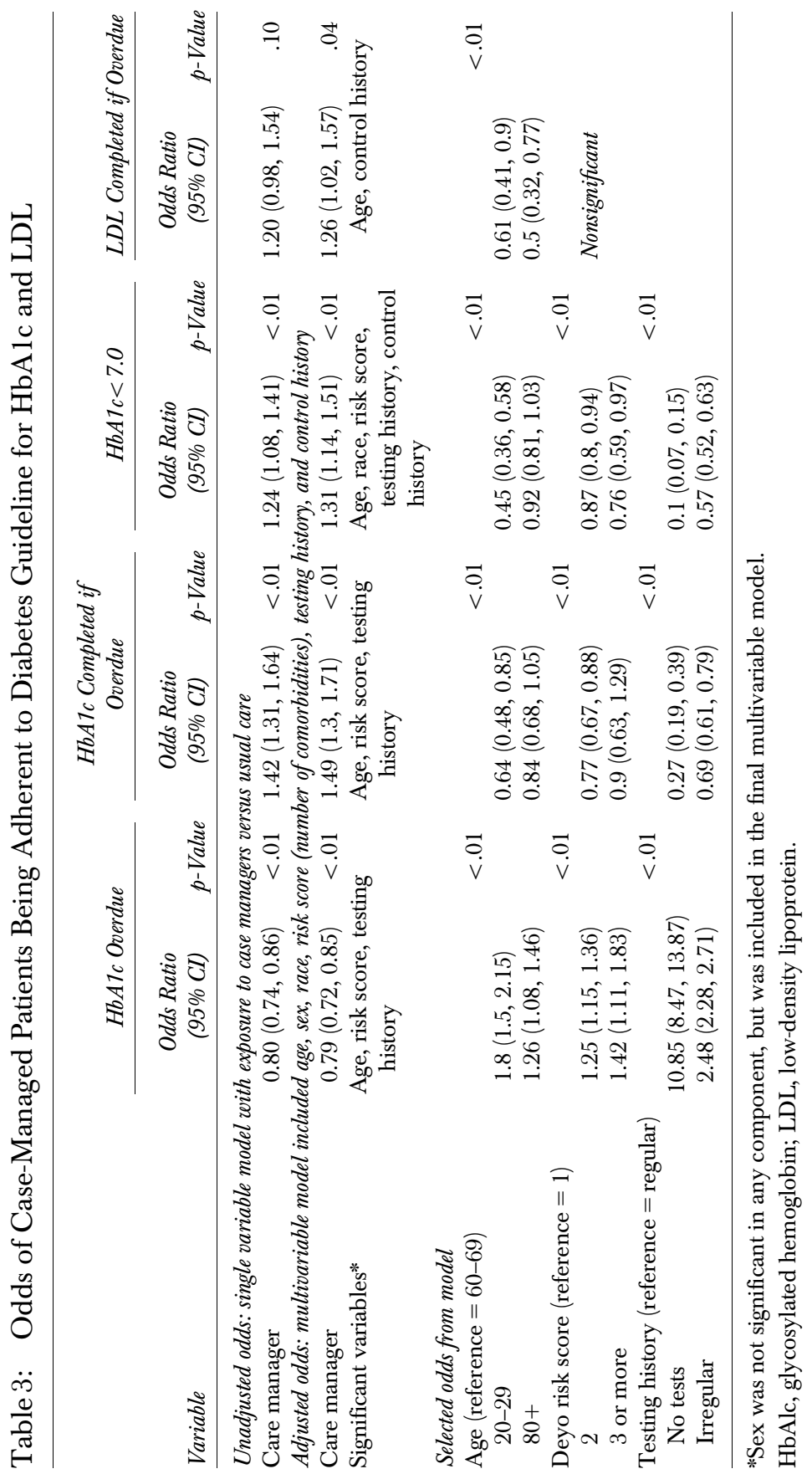


regularity, age (with the very young and the very old at higher risk of being overdue), and increasing comorbidity index score. The group of patients whose past testing was irregular or nonexistent had 2.5 and 10.9 times the odds of being overdue, respectively, versus patients in whom testing had been regular (no previous tests: OR 10.85, 95 percent CI 8.47-13.87; irregular testing: OR 2.48, 95 percent CI 2.28-2.71; $p=<.01$ ).

Exposure to care managers significantly increased the odds of completing the testing once the patient was overdue for $\mathrm{HbAlc}$ (OR 1.49; 95 percent CI 1.3-1.71) and LDL (OR 1.26; 95 percent CI 1.02-1.57) testing, as seen in Table 3. Patients in the exposure and control groups with age between 20 and 29 (younger) and older than 80 years (very old), higher risk patients, and those with an irregular testing history had worse odds of being tested when overdue for HbA1c. Being of younger and very old age also decreased the odds of being tested for LDL by $40-50$ percent.

Table 4 compares the absolute and relative differences in HbAlc and LDL levels between the care-managed (exposure) and control groups. The average changes between the initial and final levels for $\mathrm{HbAlc}$ and final levels of LDL were significantly lower for the exposure group as compared with the control group (as shown in Table 4). Despite matching on history of glycemic control, the initial level of $\mathrm{HbAlc}$ in the exposure group was 0.25 percentage units higher than that of the matched controls. The correlation $(r)$ between pretest and posttest was 0.64 , and the maximum amount of decline in $\mathrm{HbA} 1 \mathrm{c}$ levels because of regression from the mean is expected to be 0.09 percent $\mathrm{HbAlc}$ greater in the exposure than the control group; the 0.09 percent is subtracted from the exposure groups' difference. The odds of the HbAlc being in the controlled range were also significantly higher for the exposure group (Table 3; OR 1.19, 95 percent CI 1.10-1.28). Again, younger age, higher risk, and irregular testing history all lowered the odds of being in control; a history of being uncontrolled or being of nonwhite or unknown race also lowered the odds that the current test result demonstrated control. No significant difference was seen between the two groups for odds of LDL below $100 \mathrm{mg} / \mathrm{dl}$.

\section{DISCUSSION}

This study demonstrates a statistically significant improvement in adherence to diabetic guidelines when generalist care managers with enhanced computer support are involved in the care of people with diabetes as compared with 


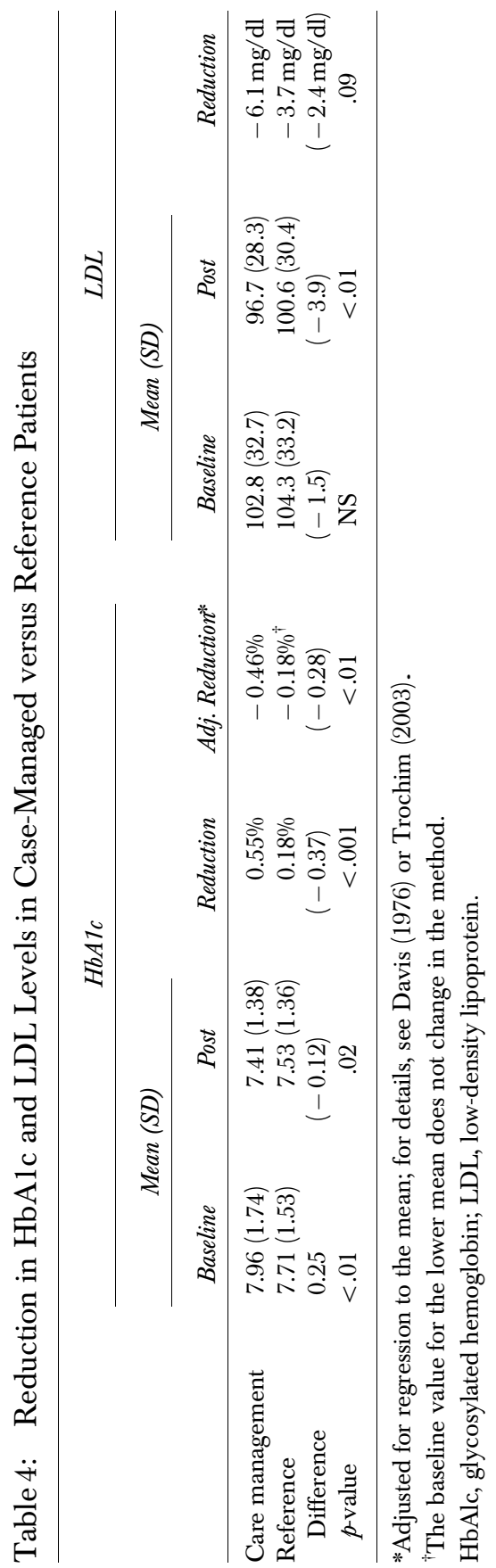


usual care-including computer support-for matched controls. In addition, the average values for LDL and HbAlc were ultimately lower for the caremanaged group versus the controls, and the odds of having glycemic control were higher in the care-managed group. These improvements, if sustained, are predicted by previous studies to lead to a $15-20$ percent reduction in complications (Viberti 2003; Vinik and Vinik 2003). A notable exception to these positive results were the very old (age 80 and older), who were less likely to achieve adherence to the guidelines at each step. These results are tempered by the nature of our effectiveness study, which makes it difficult to measure individual components of the intervention.

Evaluating our generalist care management system on a set of patients with diabetes - an expensive, complicated chronic disease with effective therapies-is an important component of the overall success of the system, especially given that patients with diabetes were only approximately 26 percent of the patients treated. Several recent studies demonstrate the differences between this study design and the current literature (Pan et al. 1997; Tuomilehto et al. 2001; Knowler et al. 2002; New et al. 2003; Taylor et al. 2003; The California Medi-Cal Type 2 Diabetes Study Group 2004). Most used an idealized trial format, with separate diabetes clinics, endocrinologists, and/or nurse specialists to improve out-of-control blood pressure and lipid levels in people with diabetes. These investigators have been able to show improvement in control, adherence, and even mortality of diabetics randomized to treatment clinics versus control. The effectiveness format in the present study uses a different implementation method. No specialized clinics were created; rather, the usual day-to-day activities of PCPs were augmented by the presence of the generalist care managers in a team-based approach. Wagner et al. (2001) conducted trials with both frail geriatric and diabetic patients using chronic care clinics that were closer to our approach (although still disease specific). Unlike the current study, the diabetic arm of Wagner's study showed improvement in adherence but no change in HbAlc in the intervention $(N=278)$ versus control patients (Wagner et al. 2001).

Our approach has a strong basis in theory; the care managers receive training in and apply the stages of change model (Prochaska 2003), the coaching model (Koenigsberg, Bartlett, and Cramer 2004), and Wagner's CCM (Wagner et al. 2001), as they care for patients with a variety of illnesses. Our implementation of the CCM is different from most, however, in that it adds several aspects of information system components. The care managers and other team members have access to and use multiple features of a shared electronic medical record, specifically a summarized, structured form with 
patient-specific results; the success of the team approach with generalist care managers may indeed depend on this distributed, longitudinal technology, which enables the team to apply multiple guidelines with ease. Components that facilitate this process included the ability to access the entire patient record easily, specific decision support mechanisms that help them to efficiently address the needs of a population by providing lists of alerts for patients who require attention, and patient-specific electronic communication. Importantly, this intervention allows for smoother integration into the primary care workflow, as information technology helps facilitate communication and the application of multiple disease guidelines and other resources in a single visit rather than the creation of specialized clinics or additional visits for other comorbidities. Studies indicate that this is an important factor in the inefficiency of primary care clinics (Flocke, Frank, and Wenger 2001; Rothman and Wagner 2003). In addition, the generalist approach may allow the care managers to focus more on the needs of the patient than the needs of one particular disease, improving patient-centric care delivery and prioritizing care delivery (Allen 1994; Crystal et al. 1999; Naylor et al. 1999; Bull et al. 2000). Finally, this generalist implementation had all of the elements of the CCM, including health care organization, self-management support, clinical information systems, decision support, connection to the community, and delivery system redesign. We focused on delivery system redesign with team care and information system support; a previous study found the care delivery design to be the most influential component (Sperl-Hillen et al. 2004) but the information technology element included in our implementation is very broad and may contribute significantly to the success of our program, as described by $\mathrm{Ca}-$ salino et al. (2003).

Several potential biases exist in this study. First, referral bias may create differences between this population and other pertinent populations. Although attempts were made to match control and exposure variables based on available pertinent variables and risk factors, there are possibly other factors that would contribute to the effect seen in this paper. While it is possible that the patients who did not receive care management were in some way different from those who did, the matching variables were chosen to ensure similar previous outcomes and baseline probability of adherence and control of diabetes. Also, utilization was matched by determining the eligibility of controls based on previous visit history. Most biases in referral for this system (patients who are more ill or more difficult to control, for example) would favor a result of no differences between the groups. The higher baseline HbA1c confirms the direction of these biases; the correction for regression to the mean provides 
an appropriate perspective given this bias. The inclusion of both Type I (estimated at $<5$ percent of the study population) and Type II diabetes as well as a broad range of ages indicates a number of potential different subpopulations who might have very different treatment recommendations. To account for these different subpopulations, the chosen process and control measures are the same in the various guidelines that cover these groups, while the differing treatment recommendations were largely excluded from analysis. Thus, recommendations exist to measure LDL in even the youngest groups with diabetes, but treatment recommendations differ. Adjusting for age and estimated Type I diabetics did not affect the significance of the results. Another source of bias was the initial selection of HbAlc 7.2 percent as the cutoff for control; at the start of the study, and this was the internal guideline at the health system under study; it was selected during a period when external guidelines were shifting from 8.0 to 7.0 percent as the goal. The results do not differ with control criteria set at 7.0 or 7.2 percent; for generalizability, 7.0 percent is given in the results.

Biases based on environmental variables, such as clinic milieu or other provider care, were minimized by including a large proportion of control patients who were seen in the same or similar clinics or by the same physicians but not referred. Biases as a result of information system effects were also minimized by ensuring that all clinicians included had access to and generally used the clinical information system. The effect of individual components of the care management system described in this study is difficult to disambiguate because of the study design. For instance, five of the care managers were not certified diabetic educators; although a separate analysis indicates that outcomes did not differ in these five care managers, the relative role of diabetic education is difficult to discern. All patients had access to diabetes education through groups and individual educators, but the kind, amount, and quality of diabetic education may differ; part of this difference is as a result of the design of the system, however. Finally, some of the improvements may be because of the increased scrutiny of these patients (Hawthorne effect) and could attenuate over time.

The averaged difference in HbAlc and LDL between the groups is consistent with previous studies, despite the fact that both the initial and final $\mathrm{HbA1c}$ levels of both our groups were lower than most studies. Many patients with out-of-control HbAlc and LDL levels were likely excluded from the control group in this study because they were never tested (substantiated by the data on irregular and unknown testing) - the effect of care manager is likely underestimated because of this difficulty in study design. 
It would be important to study the persistence of this effect through a long-term prospective study to determine assessment of the reasons for referral and overall satisfaction with the system. The independent effect of computer assistance and other intervention components is also of interest; technological assistance likely contributes to the ability of the care managers to positively impact patient outcomes by facilitating access to patient data relevant to multiple guideline compliance, by meeting specific information needs of care managers, and through the messaging abilities of the system. In effectiveness studies such as this one, the generalizability of the intervention arm and the comparability of the control arm are important. In this study, the information system components are more advanced than many other systems; however, the improvement beyond the information systems indicates that such systems are not enough: a broader care management system can further improve care. As a significant problem in health care delivery is the inadequate application of known treatments for chronic diseases and most patients with chronic diseases received their health care from primary care providers, models that can improve adherence to guidelines of care in this setting are important (Glasgow, Vogt, and Boles 1999; Rothman and Wagner 2003). Overall, this study represents an important first step in evaluating a generalist multidisease care management program in a real-world setting.

\section{REFERENCES}

AACE. 2000. AACE Medical Guidelines for Clinical Practice for the Diagnosis and Treatment of Dyslipidemia and Prevention of Atherogenesis." Endocrine Practice 6 (2): 162-213.

ADA. 2003. Standards of Medical Care for Patients with Diabetes Mellitus." Diabetes Care 26 (suppl 1): S33-50.

AHM. 2001. "Glossary" [accessed on 23 October, 2001]. Available at http:// www.academyforhealthcare.com/glossary/

Allen, S. A. 1994. "Medicare Case Management." Home Healthcare Nurse 12 (3): 21-7.

American Diabetes Association. 2003. "Diabetes Fact Sheet" [accessed on 7/31, 2003]. Available at http://www.diabetes.org/main/info/facts/facts_natl.jsp

Bodenheimer, T., E. H. Wagner, and K. Grumbach. 2002a. "Improving Primary Care for Patients with Chronic Illness.” Journal of the American Medical Association 288 (14): 1775-9.

- 2002b. "Improving Primary Care for Patients with Chronic Illness: The Chronic Care Model, Part 2." Journal of the American Medical Association 288 (15): 1909-14. 
Bond, G. R., L. D. Miller, R. D. Krumwied, and R. S. Ward. 1988. "Assertive Case Management in Three CMHCs: A Controlled Study." Hospital and Community Psychiatry 39 (4): 411-8.

Bull, M. J., H. E. Hansen, and C. R. Gross. 2000. "A Professional-Patient Partnership Model of Discharge Planning with Elders Hospitalized with Heart Failure.” Applied Nursing Research 13 (1): 19-28.

Casalino, L., R. R. Gillies, S. M. Shortell, J. A. Schmittdiel, T. Bodenheimer, J. C. Robinson, T. Rundall, N. Oswald, H. Schauffler, and M. C. Wang. 2003. "External Incentives, Information Technology, and Organized Processes to Improve Health Care Quality for Patients with Chronic Diseases." Journal of the American Medical Association 289 (4): 434-41.

Casalino, L. P. 2003. "Markets and Medicine: Barriers to Creating a 'Business Case for Quality'.” Perspectives in Biology and Medicine 46 (1): 38-51 (discussion 52-4).

CMSA. 2003. "Best Practices in Physician and Case Management Collaboration to Improve Patient Care.” Consensus Paper of the 2003 Physician and Case Management Summit.

Crystal, S., A. T. Lo Sasso, and U. Sambamoorthi. 1999. "Incidence and Duration of Hospitalizations among Persons with AIDS: An Event History Approach." Health Services Research 33 (6): 1611-38.

Davis, C. E. 1976. "The Effect of Regression to the Mean in Epidemiologic and Clinical Studies." American Journal of Epidemiology 104 (5): 493-8.

D'Ercole, A., E. Struening, J. L. Curtis, E. J. Millman, and A. Morris. 1997. "Effects of Diagnosis, Demographic Characteristics, and Case Management on Rehospitalization.” Psychiatric Services 48 (5): 682-8.

Deyo, R. A., D. C. Cherkin, and M. A. Ciol. 1992. "Adapting a Clinical Comorbidity Index for Use with ICD-9-CM Administrative Databases." Journal of Clinical Epidemiology 45 (6): 613-9.

Duran, L. S. 2003. "Motivating Health: Strategies for the Nurse Practitioner." Journal of the American Academy of Nurse Practitioners 15 (5): 200-5.

Engelgau, M. M., L. S. Geiss, J. B. Saaddine, J. P. Boyle, S. M. Benjamin, E. W. Gregg, E. F. Tierney, N. Rios-Burrows, A. H. Mokdad, E. S. Ford, G. Imperatore, and K. M. Narayan. 2004. "The Evolving Diabetes Burden in the United States." Annals of Internal Medicine 140 (11): 945-50.

Flocke, S. A., S. H. Frank, and D. A. Wenger. 2001. "Addressing Multiple Problems in the Family Practice Office Visit." Journal of Family Practice 50 (3): 211-6.

Glasgow, R. E., T. M. Vogt, and S. M. Boles. 1999. "Evaluating the Public Health Impact of Health Promotion Interventions: The RE-AIM Framework." American Journal of Public Health 89 (9): 1322-7.

Goldstein, D. E., R. R. Little, R. A. Lorenz, J. I. Malone, D. M. Nathan, and C. M. Peterson. 2004. "Tests of Glycemia in Diabetes.” Diabetes Care 27 (suppl 1): S91-3.

Haffner, S. M. 2004. "Dyslipidemia Management in Adults with Diabetes.” Diabetes Care 27 (suppl 1): S68-71.

Huber, P. 1967. "The Behavior of Maximum Likelihood Estimates under NonStandard Conditions." In Proceedings of the Fifth Berkeley Symposium in Mathemat- 
ical Statistics and Probability, pp 221-33. Berkeley, CA: University of California Press.

Knowler, W. C., E. Barrett-Connor, S. E. Fowler, R. F. Hamman, J. M. Lachin, E. A. Walker, and D. M. Nathan. 2002. "Reduction in the Incidence of Type 2 Diabetes with Lifestyle Intervention or Metformin." Nerw England Journal of Medicine 346 (6): 393-403.

Koenigsberg, M. R., D. Bartlett, and J. S. Cramer. 2004. "Facilitating Treatment Adherence with Lifestyle Changes in Diabetes." American Family Physician 69 (2): 309-16.

Matthews, D. R. 1999. "The Natural History of Diabetes-Related Complications: The UKPDS Experience. United Kingdom Prospective Diabetes Study.” Diabetes Obesity E Metabolism 1 (suppl 2): S7-13.

McGrew, J. H., G. R. Bond, L. Dietzen, M. McKasson, and L. D. Miller. 1995. "A Multisite Study of Client Outcomes in Assertive Community Treatment." Psychiatric Services 46 (7): 696-701.

Mokdad, A. H., E. S. Ford, B. A. Bowman, W. H. Dietz, F. Vinicor, V. S. Bales, and J. S. Marks. 2003. "Prevalence of Obesity, Diabetes, and ObesityRelated Health Risk Factors." Journal of the American Medical Association 289 (1): 76-9.

Naylor, M. D., D. Brooten, R. Campbell, B. S. Jacobsen, M. D. Mezey, M. V. Pauly, and J. S. Schwartz. 1999. "Comprehensive Discharge Planning and Home Follow-Up of Hospitalized Elders: A Randomized Clinical Trial." Journal of the American Medical Association 281 (7): 613-20.

New, J. P., J. M. Mason, N. Freemantle, S. Teasdale, L. M. Wong, N. J. Bruce, J. A. Burns, and J. M. Gibson. 2003. "Specialist Nurse-Led Intervention to Treat and Control Hypertension and Hyperlipidemia in Diabetes (SPLINT): A Randomized Controlled Trial." Diabetes Care 26 (8): 2250-5.

Nicollerat, J. A. 2000. "Implications of the United Kingdom Prospective Diabetes Study (UKPDS) Results on Patient Management." Diabetes Education 26 (suppl): $8-10$.

Norris, S. L., and D. E. Olson. 2004. "Implementing Evidence-Based Diabetes Care in Geriatric Populations. The Chronic Care Model." Geriatrics 59 (6): 35-9 (quiz 40).

Pan, X. R., G. W. Li, Y. H. Hu, J. X. Wang, W. Y. Yang, Z. X. An, Z. X. Hu, J. Lin, J. Z. Xiao, H. B. Cao, P. A. Liu, X. G. Jiang, Y. Y. Jiang, J. P. Wang, H. Zheng, H. Zhang, P. H. Bennett, and B. V. Howard. 1997. "Effects of Diet and Exercise in Preventing NIDDM in People with Impaired Glucose Tolerance.” Diabetes Care 20 (4): 537-44.

Prochaska, J. O. 2003. "Staging: A Revolution in Helping People Change.” Managed Care 12 (9 suppl): 6-9.

Rothman, A. A., and E. H. Wagner. 2003. "Chronic Illness Management: What Is the Role of Primary Care?” Annals of Internal Medicine 138 (3): 256-61.

Spencer, L., F. Pagell, M. E. Hallion, and T. B. Adams. 2002. "Applying the Transtheoretical Model to Tobacco Cessation and Prevention: A Review of Literature." American Journal of Health Promotion 17 (1): 7-71. 
Sperl-Hillen, J. M., L. I. Solberg, M. C. Hroscikoski, A. L. Crain, K. I. Engebretson, and P. J. O'Connor. 2004. "Do All Components of the Chronic Care Model Contribute Equally to Quality Improvement?" Joint Commission Journal on Quality and Safety 30 (6): 303-9.

Taylor, C. B., N. H. Miller, K. R. Reilly, G. Greenwald, D. Cunning, A. Deeter, and L. Abascal. 2003. "Evaluation of a Nurse-Care Management System to Improve Outcomes in Patients with Complicated Diabetes." Diabetes Care 26 (4): 1058-63.

The California Medi-Cal Type 2 Diabetes Study Group. 2004. "Closing the Gap: Effect of Diabetes Case Management on Glycemic Control among Low-Income Ethnic Minority Populations: The California Medi-Cal Type 2 Diabetes Study." Diabetes Care 27 (1): 95-103.

Toth, E. L., S. R. Majumdar, L. M. Guirguis, R. Z. Lewanczuk, T. K. Lee, and J. A. Johnson. 2003. "Compliance with Clinical Practice Guidelines for Type 2 Diabetes in Rural Patients: Treatment Gaps and Opportunities for Improvement." Pharmacotherapy 23 (5): 659-65.

Trochim, W. M. 2003. "The Research Methods Knowledge Base: The Effect of Regression to the Mean" [accessed on Sept. 11, 2003]. Available at http://trochim. human.cornell.edu/kb/index.htm

Tuomilehto, J., J. Lindstrom, J. G. Eriksson, T. T. Valle, H. Hamalainen, P. IlanneParikka, S. Keinanen-Kiukaanniemi, M. Laakso, A. Louheranta, M. Rastas, V. Salminen, and M. Uusitupa. 2001. "Prevention of Type 2 Diabetes Mellitus by Changes in Lifestyle among Subjects with Impaired Glucose Tolerance." New England Journal of Medicine 344 (18): 1343-50.

Turner, R. C., C. A. Cull, V. Frighi, and R. R. Holman. 1999. "Glycemic Control with Diet, Sulfonylurea, Metformin, or Insulin in Patients with Type 2 Diabetes Mellitus: Progressive Requirement for Multiple Therapies (UKPDS 49)." Journal of the American Medical Association 281 (21): 2005-12.

Viberti, G. 2003. "The Need for Tighter Control of Cardiovascular Risk Factors in Diabetic Patients." Journal of Hypertension 21 (suppl 1): S3-6.

Vinik, A. I., and E. Vinik. 2003. "Prevention of the Complications of Diabetes." American Journal of Managing Care 9 (3 suppl): S63-80 (quiz S81-4).

Wagner, E. H., L. C. Grothaus, N. Sandhu, M. S. Galvin, M. McGregor, K. Artz, and E. A. Coleman. 2001. "Chronic Care Clinics for Diabetes in Primary Care: A System-Wide Randomized Trial.” Diabetes Care 24 (4): 695-700.

White, H. 1982. "Maximum Likelihood Estimation of Misspecified Models." Econometrica 50: 1-25. 